\title{
Multiple Liver Abscesses Caused by Streptococcus constellatus: A Case Report
}

\author{
Nobuhiro Takeuchi, Hiromichi Naito, Tetsuya Yumoto, ${ }^{2}$ Kohei Tsukahara, ${ }^{2}$ Taihei Yamada, \\ Takaaki Osako,2,Junichi Soneda, and Atsunori Nakao²
}

\begin{abstract}
Background: Pyrogenetic liver abscess is often caused by gram-negative bacilli, including Escheria coli or Klebsiella pneumoniae, but rarely by Streptococcus constellatus. Streptococcus constellatus is a commensal of the oral cavity, respiratory tract system, intestine, and urogenital organs. Herein, we report a case of multiple liver abscesses caused by Streptococcus constellatus.

Case Presentation: A 66-year-old male presented with a high-grade fever with no known source. Laboratory analysis revealed severely elevated inflammation levels, moderately increased liver and biliary enzymes, and moderately worsened renal function. Sonazoid ${ }^{\circledR}$ (GE Healthcare AS, Oslo, Norway)-enhanced sonography revealed marginally contrast-enhanced masses with a non-contrast-enhanced center. On the basis of these results, the patient was diagnosed with multiple liver abscesses. Subsequently, meropenem $1 \mathrm{~g} / \mathrm{d}$ was prescribed. On day two, percutaneous abscess drainage was performed. Culture from abscess drainage and blood culture revealed the presence of Streptococcus constellatus. Because of antibiotic sensitivity, meropenem was replaced by piperacillin $6 \mathrm{~g} / \mathrm{d}$. Clinical course was uneventful and the patient was discharged on day 53 .

Conclusion: The pathogenic potential of Streptococcus constellatus has been recognized recently. Although the Streptococcus milleri group has been focused on primarily for its commensal nature, clinicians need to be aware of its pathogenic nature and biologic character of forming liver abscesses.
\end{abstract}

Keywords: liver abscesses; sonazoid-enhanced sonography; Streptococcus constellatus

A LTHOUGH GRAM-NEGATIVE BACILLI, including Escherichia coli and Klebsiella pneumoniae, are well recognized as causing pyogenic liver abscesses, the Streptococcus milleri group, comprising Streptococcus intermedius, Streptococcus constellatus, and Streptococcus anginosus, are not well-known as causative bacteria of liver abscesses. The Streptococcus milleri group has been considered a commensal micro-organism in the oral cavity, respiratory tract system, intestine, and urogenital system, therefore, the pathogenic potential of the Streptococcus milleri group has been disregarded. As far as we know, only a few cases of pyogenic liver abscess caused by the Streptococcus milleri group have been reported [1-3]. Herein, we report a case of multiple liver abscesses caused by Streptococcus constellatus and discuss its pathogenic potential and aggressive nature based on the literature.

\section{Case Presentation}

A 66-year-old male visited our department by ambulance with a fever of unknown cause that had lasted for seven days. His past medical history included Parkinson disease that had been treated with levodopa, entacapone, ropinirole, and istradefylline. He was observed for a while, but the fever did not ameliorate. He experienced chills and a high-grade fever of $40.0^{\circ} \mathrm{C}$. He could not walk without assistance.

Physical examination upon arrival revealed a Glasgow Coma Scale of 11; blood pressure, $131 / 72 \mathrm{~mm} \mathrm{Hg}$; heart rate,

\footnotetext{
${ }^{1}$ Department of Internal Medicine, Kobe Tokushukai Hospital, Tarumi, Kobe, Japan

${ }^{2}$ Department of Critical Care and Emergency Medicine, Okayama University Hospital, Okayama, Japan.

(C) Nobuhiro Takeuchi et al. 2017; Published by Mary Ann Liebert, Inc. This Open Access article is distributed under the terms of the Creative Commons License (http://creativecommons.org/licenses/by/4.0), which permits unrestricted use, distribution, and reproduction in any medium, provided the original work is properly credited.
} 
95 beats per minute with regular rhythm; blood oxygen saturation under atmospheric conditions, $91 \%$; and body temperature, $40^{\circ} \mathrm{C}$. The abdomen was slightly distended with normal peristalsis with mild tenderness in the epigastric area. No mass was palpable and no signs of peritoneal irritation were observed over the entire abdomen. Oral inspection revealed decayed teeth as well as several defective teeth.

Blood analyses revealed leukocytosis (17,000 white blood cells per microliter with $92.0 \%$ neutrophils) and severely decreased platelet counts $\left(9 \times 10^{4}\right.$ per microliter). Blood chemistry revealed $28.47 \mathrm{mg} / \mathrm{dL}$ C-reactive protein, mild hyponatremia $(136 \mathrm{mEq} / \mathrm{mL})$ and hypokalemia $(3.0 \mathrm{mEq} / \mathrm{mL})$, moderately impaired renal function $(2.49 \mathrm{mg} / \mathrm{dL}$ creatinine and $45.5 \mathrm{mg} / \mathrm{dL}$ blood urea nitrogen), and coagulant dysfunction (37\% prothrombin; $47.0 \mathrm{sec}$ activated partial thromboplastin time; $80 \%$ antithrombin III; $59 \mathrm{mg} / \mathrm{mL}$ fibrin/fibrinogen degradation products (FDP), and $30.1 \mathrm{mg} / \mathrm{mL}$ D-dimer). Furthermore, moderately decreased total protein $(5.7 \mathrm{~g} / \mathrm{dL})$ and albumin $(2.7 \mathrm{~g} / \mathrm{dL})$ and mildly increased total bilirubin $(2.0 \mathrm{mg} / \mathrm{dL})$; gamma-glutamyltransferase (177 IU/L); alkaline phosphatase (464 IU/L); aspartate aminotransferase (172 IU/L); alanine transaminase $(308 \mathrm{IU} / \mathrm{L})$; and lactate dehydrogenase (332 IU/l) were also revealed.
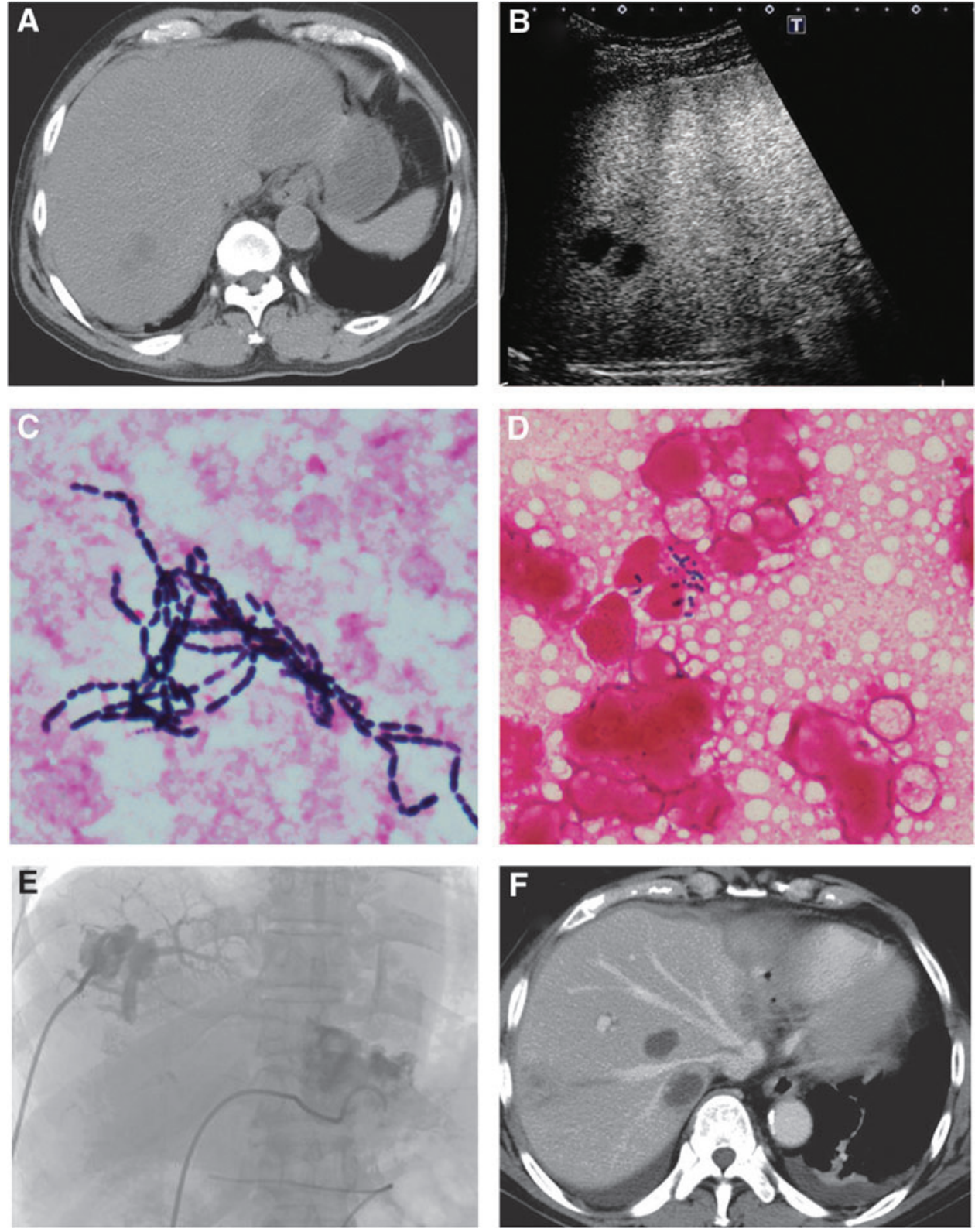

FIG. 1. (A) Non-contrast computed tomography at arrival reveals multiple low-density masses in the liver, including a $57 \times 43 \mathrm{~mm}$ mass at S1 and a $23 \times 26 \mathrm{~mm}$ abscess at S6. (B) Contrast-enhanced ultrasonography with Sonazoid ${ }^{\circledR}$ (GE Healthcare AS, Oslo, Norway) reveals un-enhanced areas. The result is compatible with liver abscess. (C) Abscess culture reveals the presence of Streptococcus constellatus. (D) Blood culture reveals the presence of Streptococcus constellatus. (E) Contrast injection from a pigtail catheter reveals relation between abscess and portal vein. (F) Contrast-enhanced computed tomography reveals multiple low-density masses with contrast-enhanced margins. The result is compatible with liver abscesses. 
Electrocardiography revealed a normal, regular heart rhythm without ST changes. Chest radiograph revealed no cardiomegaly, pleural effusions, or pulmonary congestion. Non-contrast abdominal computed tomography (CT) revealed multiple masses in the entire liver (Fig. 1A). Contrastenhanced CT was not performed because of the patient's deteriorated renal function. Sonazoid ${ }^{\circledR}$ (GE Healthcare AS, Oslo, Norway)- enhanced sonography was performed, revealing marginally enhanced masses with non-contrasted lesions in the center (Fig. 1B). On the basis of these results, the patient was diagnosed with multiple liver abscesses; meropenem $1 \mathrm{~g} / \mathrm{d}$ was then administered. On day two, percutaneous abscess drainage (PTAD) was performed. A 7F pigtail catheter was placed in a $57 \times 43 \mathrm{~mm}$ abscess at $\mathrm{S} 1$ of the liver. Samples from abscess drainage and blood culture revealed the presence of Streptococcus constellatus (Fig. 1C, abscess culture and Fig. 1D, blood culture). Because of antibiotic sensitivity, meropenem was replaced by piperacillin $6 \mathrm{~g} / \mathrm{d}$.

On day three, oxygen inhalation was required at $8 \mathrm{~L} / \mathrm{min}$ with a face mask because of the patient's worsening respiratory condition. Moreover, emergency hemodialysis was introduced to treat his oliguria and deteriorating renal function. On day six, additional PTAD was performed and a 7F pigtail catheter was inserted to a $23 \times 26 \mathrm{~mm}$ abscess at $\mathrm{S} 6$ of the liver. Contrast agent from the drainage tube revealed the relation between the abscess and portal vein (Fig. 1E). On day 10 , contrast-enhanced CT was performed after the patient's renal function improved by returning to a normal range and revealed multiple masses with contrast margins (Fig. 1F); the result was compatible with liver abscess. After inflammatory reactions improved, endoscopic retrograde cholangiopancreatography was performed to investigate bile duct abnormalities; none were found. Colonoscopy was performed to evaluate the origin of the infection and revealed multiple diverticula in the ascending colon. Clinical course was uneventful and the patient discharged on day 53 .

\section{Discussion}

Causative microbes of pyogenic liver abscesses are commonly E. coli or Klebsiella pneumoniae; the Streptococcus milleri group has been recognized only rarely to cause liver abscesses. The pathogenic potential of the Streptococcus milleri group has been underestimated for a long time because of its commensal nature [4]. Recently, with the development of microbiologic techniques for detecting the Streptococcus milleri group, cases of Streptococcus milleri group infections have been reported increasingly. However, except for a few reports, liver abscess cases caused by the Streptococcus milleri group have been reported rarely thus far [1-3].

When causative agents of liver abscesses are unknown, cefem antibiotics are chosen to target E. coli or Klebsiella pneumoniae. The Streptococcus milleri group is less sensitive to first- and second-generation cefem antibiotics. Medical conditions related to liver abscesses caused by the Streptococcus milleri group occasionally may deteriorate with individual use of an antibiotic drug because the Streptococcus milleri group sometimes causes mixed infections. In our case, liver abscesses were caused by a single Streptococcus constellatus infection. Patients with altered immune responses, including malignancies, tend to be affected by mixed infections [5].
Liver abscesses can originate from several possible infection routes, including via the bile duct, portal vein, and hepatic artery. Among these routes, bile duct infection is the most common. Liver abscesses through the bile duct route form via retrograde infection from the bile duct to the liver resulting from the spread of bacteria from a narrowed bile duct, caused by conditions including gallbladder stones, common bile duct stones, and pancreatic or biliary malignancies. On the other hand, liver abscess from the portal vein route may occur from inflammation of the gastrointestinal system, including appendicitis, diverticulitis, or intraabdominal infections after gastric resection or colorectal resection. A recent retrospective cohort study shows that the incidence of pyogenic liver abscesses is 2.44 times higher in patients with colonic diverticular diseases than those without diverticular disease, diverticulosis (2.26), and diverticulitis (1.98) [6]. Because the Streptococcus milleri group belongs to the normal intestinal flora, damage to the intestinal mucosa plays an initial role in disseminating the microbe via the portal blood stream, leading to the formation of a liver abscess [7]. In our case, the origin of infection was likely from the existence of diverticulosis.

Literature concerning the characteristic differences between liver abscesses caused by the Streptococcus milleri group and those caused by Klebsiella spp. focuses on the aggressive nature of Streptococcus milleri group and the higher rates of bacteremia, septic shock, disseminated intravascular coagulation, metastatic dissemination, and acute renal and respiratory failure [8].

Conventionally, diagnosis of liver abscess has been made using contrast-enhanced CT. However, the use of contrast agents may increase the risk of contrast nephropathy for patients with renal dysfunction. Sonazoid can be used safely for patients with kidney failure because of its minor risk for renal damage. For patients with renal dysfunction, contrastenhanced ultrasonography with Sonazoid can be substituted with contrast-enhanced CT [9].

\section{Conclusion}

We reported a case of multiple liver abscesses caused by Streptococcus constellatus. Notably, liver abscesses resulting from the Streptococcus milleri group may present with severe critical conditions, including sepsis and renal and respiratory failure. The Streptococcus milleri group occasionally causes mixed infections, therefore, the appropriate choice of antibiotics is paramount. Although the Streptococcus milleri group has been focused on primarily for its commensal nature, clinicians need to be aware of its pathogenic nature and biologic character of forming liver abscesses.

\section{Acknowledgments}

No funding support was given for this study.

\section{Author Disclosure Statement}

No competing financial interests exist.

\section{References}

1. Rodrigues AL, Soares MC, Ramos FL, et al. Multiple pyogenic liver abscesses caused by Streptococcus constellatus in the Amazon region. Case report. Ann Hepatol 2009;8: 255-257. 
2. Gharib SD, Berger DL, Choy G, Huck AE. Case records of the Massachusetts General Hospital. Case 21-2015. A 37year-old American man living in Vietnam, with fever and bacteremia. N Engl J Med 2015;373:174-183.

3. Akuzawa N, Hatori T, Kitahara Y, Kurabayashi M. Multiple liver abscesses and bacteremia caused by Streptococcus constellatus infection: A case report. Clin Case Rep 2016;5: $69-74$.

4. Asam D, Spellerberg B. Molecular pathogenicity of Streptococcus anginosus. Mol Oral Microbiol 2014;29:145 - 155.

5. Miike T, Yamamoto S, Tahara Y, et al. A case report of gastrointestinal stromal tumor of the small intestine presenting with liver abscesses caused by Streptococcus constellatus. [in Japanese]. Nihon Shokakibyo Gakkai Zasshi 2014; $111: 1376-1383$.

6. Tsai MS, Lee HM, Hsin MC, et al. Increased risk of pyogenic liver abscess among patients with colonic diverticular diseases: A nationwide cohort study. Medicine (Baltimore) 2015;94:e2210.

7. Paraskeva KD, Bury RW, Isaacs P. Streptococcus milleri liver abscesses: An unusual complication after colonoscopic removal of an impacted fish bone. Gastrointest Endosc 2000; $51: 357-358$.

8. Law ST, Kong Li MK. Is there any difference in pyogenic liver abscess caused by Streptococcus milleri and Klebsiella spp?: Retrospective analysis over a 10 -year period in a regional hospital. J Microbiol Immunol Infect 2013;46:11 - 18 .

9. Kishina M, Koda M, Tokunaga S, et al. Usefulness of contrast-enhanced ultrasound with Sonazoid for evaluating liver abscess in comparison with conventional B-mode ultrasound. Hepatol Res 2015;45:337-342.

Address correspondence to: Dr. Atsunori Nakao

Department of Emergency and Critical Care Medicine Okayama University Graduate School of Medicine Dentistry and Pharmaceutical Sciences 2-5-1 Shikata-cho, Kita-ku Okayama-shi, Okayama, 700-8558 Japan

E-mail: qq-nakao@okayama-u.ac.jp

$$
\begin{aligned}
& \text { Abbreviations Used } \\
& \mathrm{CT}=\text { computed tomography } \\
& \mathrm{FDP}=\text { fibrin/fibrinogen degradation products } \\
& \mathrm{PTAD}=\text { percutaneous abscess drainage }
\end{aligned}
$$

Cite this article as: Takeuchi $\mathrm{N}$, Naito $\mathrm{H}$, Yumoto $\mathrm{T}$, Tsukahara K, Yamada T, Osako T, Soneda J, Nakao A (2017) Multiple liver abscesses caused by Streptococcus constellatus: A case report. Surgical Infections Case Reports 2:1, 61-64, DOI: 10.1089/crsi.2017.0013 